\title{
Risk Factors for Retinopathy of Prematurity in the Netherlands: A Comparison of Two Cohorts
}

\author{
Kasia Trzcionkowska ${ }^{a}$ Floris Groenendaal ${ }^{b}$ Peter Andriessen ${ }^{c}$ Peter H. Dijk ${ }^{d}$ \\ Frank A.M. van den Dungen ${ }^{\mathrm{e}}$ Jacqueline L. van Hillegersberg ${ }^{f}$ Sanne Koole $^{g}$ \\ René F. Kornelisse $^{\text {h }}$ Elke van Westering-Kroon ${ }^{i}$ Jeanette S. von Lindern ${ }^{j}$ \\ Clemens B. Meijssen ${ }^{k}$ Frank A.B.A. Schuerman' Katerina Steiner ${ }^{m}$ \\ Minke W.G. van Tuyl ${ }^{\mathrm{a}}$ Ruben S.G.M. Witlox ${ }^{a}$ Nicoline E. Schalij-Delfos ${ }^{a}$ \\ Jacqueline U.M. Termote ${ }^{b}$ \\ aLeiden University Medical Center, Leiden, The Netherlands; 'bilhelmina Children's Hospital, Utrecht,

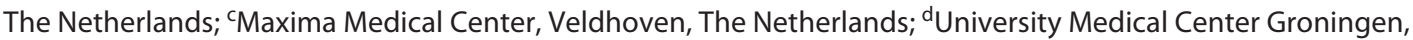 \\ Groningen, The Netherlands; ${ }^{\mathrm{e} A m s t e r d a m ~ U n i v e r s i t y ~ M e d i c a l ~ C e n t e r, ~ A m s t e r d a m, ~ T h e ~ N e t h e r l a n d s ; ~}{ }^{\mathrm{f} S t}$. Antonius \\ Hospital, Nieuwegein, The Netherlands; 9Perined, The Netherlands Perinatal Registry, Utrecht, The Netherlands; \\ hErasmus Medical Center Sophia Children's Hospital, Rotterdam, The Netherlands; 'Maastricht University Medical \\ Center+, Maastricht, The Netherlands; jGroene Hart Hospital, Gouda, The Netherlands; ${ }^{k}$ Meander Medical Center, \\ Amersfoort, The Netherlands; 'Isala Hospital, Zwolle, The Netherlands; mRadboud University Medical Center, \\ Nijmegen, The Netherlands
}

\section{Keywords}

Retinopathy of prematurity · Prematurity and screening

\begin{abstract}
Introduction: Retinopathy of prematurity (ROP) remains an important cause for preventable blindness. Aside from gestational age (GA) and birth weight, risk factor assessment can be important for determination of infants at risk of (severe) ROP. Methods: Prospective, multivariable risk-analysis study (NEDROP-2) was conducted, including all infants born in 2017 in the Netherlands considered eligible for ROP screening by pediatricians. Ophthalmologists provided data of screened infants, which were combined with risk factors from the national perinatal database (Perined). Clinical data
\end{abstract}

karger@karger.com www.karger.com/neo

Karger $\stackrel{\text { ' }}{5}$

GOPEN ACCESS
(C) 2021 The Author(s)

Published by S. Karger AG, Basel

This is an Open Access article licensed under the Creative Commons Attribution-NonCommercial-4.0 International License (CC BY-NC) (http://www.karger.com/Services/OpenAccessLicense), applicable to the online version of the article only. Usage and distribution for commercial purposes requires written permission. and potential risk factors were compared to the first national ROP inventory (NEDROP-1, 2009). During the second period, more strict risk factor-based screening inclusion criteria were applied. Results: Of 1,287 eligible infants, 933 (72.5\%) were screened for ROP and matched with the Perined data. Any ROP was found in 264 infants ( $28.3 \%$ of screened population, 2009: 21.9\%) and severe ROP (sROP) (stage $\geq 3$ ) in 41 infants (4.4\%, 2009: $2.1 \%)$. The risk for any ROP is decreased with a higher GA (odds ratio [OR] 0.59 and $95 \%$ confidence interval $[\mathrm{Cl}]$ 0.54-0.66) and increased for small for GA (SGA) $(1.73,1.11-2.62)$, mechanical ventilation $>7$ days $(2.13,1.35$ $3.37)$ and postnatal corticosteroids $(2.57,1.44-4.66)$. For sROP, significant factors were GA (OR 0.37 and $\mathrm{Cl} 0.27-0.50$ ), SGA (OR 5.65 and $\mathrm{Cl}$ 2.17-14.92), postnatal corticosteroids (OR 3.81 and $\mathrm{Cl}$ 1.72-8.40), and perforated necrotizing en-
Correspondence to:

Kasia Trzcionkowska, k.trzcionkowska@lumc.nl 
terocolitis (OR 7.55 and $\mathrm{Cl} 2.29-24.48)$. Conclusion: In the Netherlands, SROP was diagnosed more frequently since 2009. No new risk factors for ROP were determined in the present study, apart from those already included in the current screening guideline.

(c) 2021 The Author(s)

Published by S. Karger AG, Basel

\section{Introduction}

Worldwide, retinopathy of prematurity (ROP) continues to be an important cause of childhood blindness [1, $2]$. Although usually self-limiting, ROP requires treatment in approximately $8 \%$ of the overall screened population to prevent irreversible visual impairment [3]. Major risk factors of ROP are gestational age (GA) and birth weight (BW) [1]. Still, ROP is a multifactorial disorder influenced by other aspects, for example, maternal factors, medical interventions, insufficient treatment, and comorbidities [4]. Since these factors are strongly correlated to neonatal care, ROP screening should be adapted to local incidences and risk factors.

In 2009, the first nationwide inventory in the Netherlands to study ROP risk factors (NEDROP) was performed [5]. Several well-known factors were confirmed: GA, BW, length of stay (LOS) in the neonatal intensive care unit (NICU), and mechanical ventilation longer than 7 days (MV $>7$ days). Treatment with inhaled nitric oxide (iNO) was newly identified.

Subsequently, together with extensive cost-effectiveness analyses, a new ROP screening guideline was implemented in 2013 (Table 1) [6]. GA and BW were lowered, and risk factors were included (i.e., MV, sepsis, [perforated] necrotizing enterocolitis [NEC], postnatal corticosteroids, and hypotension treated with cardiotonic agents). This adjustment would allow a reduction in screened infants by $29 \%$ without missing sROP needing treatment.

Since then, several developments in Dutch neonatal care might have influenced the incidence and risk factors for ROP. First, the GA limit for active neonatal care was lowered from 25.0 to 24.0 weeks in 2010. Furthermore, following an interim meta-analysis of the NeOProM group of studies, oxygen saturation targets of NICU-admitted neonates were raised in 2014 [7]. Together with residual confounders, these changes increased the risk for ROP, which was confirmed in an inventory on ROP treatment in the Netherlands, revealing a notable increase of ROP treatment [8], from $n=57$ between 2010 and 2013 to $n=139$ between 2013 and 2016. As it has nearly been
Table 1. Inclusion criteria for ROP screening according to the previous and current Dutch guideline

\begin{tabular}{lll}
\hline Guideline & Previous (1997) & Current (2013) \\
\hline GA/BW & $<32.0$ weeks and/or & $<30.0$ weeks and/or \\
& $<1,500 \mathrm{~g}$ & $<1,250 \mathrm{~g}$ \\
Additional & - & $30.0-32.0$ weeks and/or \\
inclusion & & $1,250-1,500 \mathrm{~g}$ and presence \\
& & of $\geq 1$ risk factor \\
\hline
\end{tabular}

ROP, retinopathy of prematurity; GA, gestational age; BW, birth weight.

a decade since the first NEDROP study, the purpose of this consecutive, NEDROP-2 inventory was to determine the present risk factors associated with ROP.

\section{Methods}

Study Design

NEDROP-2, a multicenter, prospective inventory, studied infants born in 2017 and eligible for ROP screening according to the current guideline (2013), using risk-based criteria (Fig. 1).

\section{Patients and Data}

First, pediatricians reported infants eligible for ROP screening, on admission, by a set of coded data as follows: date of birth, 4 digits of the zip code, GA, BW, and when applicable, the index of multiple births $(1 / 2,2 / 2,1 / 3$, etc.). Second, ophthalmologists reported screened infants using the same code. Definitions of neonatal risk factors were identical to that of 2009 and were obtained from the national perinatal registry, Perined. Extremely low birth weight was defined as BW $<1,000 \mathrm{~g}$. Small for GA (SGA) was defined as $\mathrm{BW}<-2 \mathrm{SD}$ [9]. Oxygen exposure was defined as the number of days fully exposed to supplemental oxygen. Bronchopulmonary dysplasia was defined as need for supplemental oxygen at 36 weeks post-menstrual age. NEC was included when perforated. Sepsis was defined as clinical signs of sepsis and a positive blood culture; early and late sepsis were included. Severe intraventricular hemorrhage was classified according to the definition of Papile (stage $\geq 3$ ) [10] and cystic periventricular leukomalacia to the definition of De Vries (grade 2-3) [11]. Patent ductus arteriosus was included when treated with indomethacin, ibuprofen, paracetamol, or surgery. Hyperglycemia was defined as a blood sugar $\geq 8.0 \mathrm{mmol} / \mathrm{L}$. Longer stay at a NICU ( $>28$ days) and prolonged MV ( $>7$ days) were regarded as indicators of severe illness. Ophthalmologists documented the zone and maximum ROP stage, plus disease and ROP treatment. ROP was classified according to the International Classification of ROP and categorized according to the early treatment for ROP criteria $[12,13]$. In the Netherlands, ROP 2+ in zone II is only treated with presence of severe or progressive plus disease. For comparison with the NEDROP-1, ROP stage $1-2$ was defined as mild and stage $3-5$ as sROP. Aggressive posterior ROP and ROP in zone I with plus dis- 
Fig. 1. Population flow chart. ROP, retinopathy of prematurity. ${ }^{*}$ Perined: national perinatal registry.

\begin{tabular}{|c|c|}
\hline \multicolumn{2}{|c|}{$\begin{array}{l}\text { Live births in } 2017 \\
\quad n=165,728\end{array}$} \\
\hline \multicolumn{2}{|c|}{$\begin{array}{l}\text { Infants reported by pediatricians to be eligible for ROP screening according to the } \\
\qquad \begin{array}{l}2013 \text { screening guideline } \\
\qquad n=1,287\end{array}\end{array}$} \\
\hline \multicolumn{2}{|c|}{$\begin{array}{l}\text { NEDROP population eligible for screening and coupled with Perined* } \\
\qquad \begin{aligned} n & =1,106 \\
(1,106 / 1,287 & =85.9 \% \text { coupled })\end{aligned}\end{array}$} \\
\hline \multicolumn{2}{|c|}{$\begin{array}{l}\text { NEDROP population actually screened for ROP and coupled with Perined* } \\
\qquad n=933\end{array}$} \\
\hline \multicolumn{2}{|c|}{ ROP } \\
\hline Mild ROP $n=223$ & Severe ROP $n=41$ \\
\hline
\end{tabular}

ease were also considered sROP. In the present cohort, only 1 infant was found with aggressive posterior ROP and no infants with ROP zone I with plus disease. Based on the individual code, neonatal and ophthalmological data were merged.

\section{Statistical Analysis}

Due to differences in inclusion criteria, comparison of incidence of sROP between NEDROP-1 and NEDROP-2 was calculated by using live births as the denominator. Clinical data of infants with any degree of ROP or sROP were compared using $t$-tests or $\chi^{2}$-tests, where appropriate. A $p$ value $<0.05$ was considered significant. Multivariable analysis was performed for "any ROP" and "severe ROP" by backward analysis to reduce the final model as much as possible, using the software package "R" (https://www.r-project.org). Odds ratios and $95 \%$ confidence intervals were presented. With $>900$ infants studied and an estimated incidence of mild ROP of $20 \%$ and sROP of 3-5\%, the power of the study was $>0.90$ to examine at least 5 variables for "any ROP" and "severe ROP."

\section{Results}

Participation of all Dutch hospitals (80) involved in ROP screening was realized. Pediatricians reported 1,287 infants eligible for screening according to the 2013 guideline. Date of birth and zip code generated the highest merging rate for the NEDROP-2 database, with the perinatal registry, resulting in a combined dataset of 1,106/1,287 babies (85.9\%) (Fig. 1). Not all data could be merged because of registry errors or no record in Perined. Of the 1,106 coupled neonates, 933 (84.4\%) were actually screened for ROP. Infants were not screened due to death before first screening (103/173), transfer abroad (1/173), no show up (3/173), old criteria used (49/173), and unknown reasons (17/173). The remaining 21/173 infants (1.9\%) were falsely not screened. Median (interquartile range) overall GA and $\mathrm{BW}$ of the screened infants were 28.9, 27.3-30.3 weeks (NEDROP-1: 29.8, 28.1-31.1) and 1,150, 935-1,350 g (NEDROP-1: 1,260, 1,020-1,500), respectively. Other clinical characteristics are presented in Table 2.

ROP was found in 264 infants $(28.3 \%)$ of whom 223 (23.9\%) had mild, 41 (4.4\%) sROP, 36 (3.9\%) type 1 , and $11(1.2 \%)$ type 2 ROP. ROP was inversely associated with increasing GA and BW (Table 2). Only 5/41 infants with sROP had a GA >28.0 weeks; in none, GA was $>30.0$ weeks. All 5 were severely ill, being SGA and/or required extensive neonatal interventions (i.e., sepsis and prolonged MV).

After adjusting for GA and SGA, risk factors achieving statistical significance for any ROP were $\mathrm{MV}>7$ days and postnatal corticosteroids (Table 3 ) and for sROP, perforated NEC and postnatal corticosteroids (Table 4). Although MV $>7$ days was also significantly associated with severe ROP (odds ratio 3.00 [1.35-7.03, $p=0.008]$ ), administration of postnatal corticosteroids had higher odds for the development of severe ROP. In our final models, no interactions were found.

In Figure 2, an estimated risk of (severe) ROP is shown based on our model. It becomes clear that apart from GA, risk factors play an important role for the development of 
Table 2. Descriptive statistics

\begin{tabular}{|c|c|c|c|c|c|}
\hline Characteristics & $\begin{array}{l}\text { Total, } \\
n, \% \\
933(100)\end{array}$ & $\begin{array}{l}\text { No ROP, } \\
n, \% \\
669(71.7)\end{array}$ & $\begin{array}{l}\text { ROP stage } 1 \\
\text { or } 2, n, \% \\
223(23.9)\end{array}$ & $\begin{array}{l}\text { ROP stage } 3 \\
\text { and above, } n \text {, \% } \\
41(4.4)\end{array}$ & $p$ value \\
\hline \multirow[t]{2}{*}{ GA median (IQR) min-max } & $28.9(3.0)$ & $29.4(2.6)$ & $27.6(2.5)$ & $25.7(0.6)$ & \multirow[t]{2}{*}{$<0.001$} \\
\hline & $24.0-32.9$ & $24.0-34.9$ & $24.0-32.0$ & $24.1-30.0$ & \\
\hline BW median (IQR) min-max & $1,150(415)$ & $1,210(375)$ & $975(380)$ & $700(231)$ & $<0.001$ \\
\hline ELBW $<1,000 \mathrm{~g}$ & $293(31.4)$ & $143(21.4)$ & $114(51.1)$ & $36(87.8)$ & $<0.001$ \\
\hline SGA $p<-2$ SD & $221(23.7)$ & $163(24.4)$ & $46(20.6)$ & $12(29.3)$ & 0.362 \\
\hline Multiple births & $236(25.3)$ & $162(24.2)$ & $68(30.5)$ & $6(14.6)$ & $<0.001$ \\
\hline \multicolumn{6}{|l|}{ LOS NICU, days } \\
\hline 0 & $24(2.6)$ & $21(3.1)$ & $3(1.3)$ & 0 & $<0.001$ \\
\hline$\leq 7$ & $254(27.2)$ & $195(29.1)$ & $53(23.8)$ & $6(14.6)$ & \multirow{3}{*}{$<0.001$} \\
\hline$>7$ & $170(18.2)$ & $62(9.3)$ & $78(35.0)$ & $30(73.2)$ & \\
\hline Missing & $12(1.3)$ & $5(0.7)$ & $6(2.7)$ & $1(2.4)$ & \\
\hline \multicolumn{6}{|l|}{ Supplemental $\mathrm{O}_{2}$, days } \\
\hline 0 & $224(24.0)$ & $189(28.3)$ & $32(14.3)$ & $3(7.3)$ & \multirow[t]{4}{*}{$<0.001$} \\
\hline$\leq 28$ & $399(42.8)$ & $310(46.3)$ & $83(37.2)$ & $6(14.6)$ & \\
\hline$>28$ & $240(25.7)$ & $111(16.6)$ & $97(43.5)$ & $32(78.0)$ & \\
\hline Missing & $70(7.5)$ & $59(8.8)$ & $11(4.9)$ & 0 & \\
\hline Sepsis & $353(37.8)$ & $239(35.7)$ & $85(38.1)$ & $29(70.7)$ & $<0.001$ \\
\hline IVH stage $\geq 3$ & $48(5.1)$ & $28(4.2)$ & $15(6.7)$ & $5(12.2)$ & 0.037 \\
\hline Cystic PVL & $10(1.1)$ & $4(0.6)$ & $4(1.8)$ & $2(4.9)$ & 0.017 \\
\hline IRDS & $479(51.3)$ & $316(47.2)$ & $135(60.5)$ & $28(68.3)$ & $<0.001$ \\
\hline
\end{tabular}

All characteristics are described as absolute number $(n)$ and percentage of total (\%), except for Apgar, BW, and GA, for which median (IQR) and/or minimum to maximum values were used. Apgar 5, Apgar score 5 min after birth; BPD, bronchopulmonary dysplasia; BW, birth weight; ELBW, extremely low birth weight; GA, gestational age; iNO, inhaled nitric oxide; IRDS, infant respiratory distress syndrome; IQR, interquartile range; IVH, intraventricular hemorrhage; LOS NICU, length of stay at neonatal intensive care unit; NEC, necrotizing enterocolitis; PDA, patent ductus arteriosus; PMA, post-menstrual age; PVL, periventricular leukomalacia; SGA, small for gestational age; ROP, retinopathy of prematurity; MV, mechanical ventilation; APROP, aggressive posterior ROP. $p$ value: No ROP versus ROP stage 1 or 2, ROP stage 3, and above including APROP.

sROP in infants born $<30.0$ weeks. The difference in probability to develop any ROP between high- and lowrisk infants remains almost the same for every week of gestation. In contrast, for sROP, this difference decreases with increasing GA. Finally, for infants with a GA $<26.0$ weeks with a high-risk profile, the risk to develop any ROP is almost equal to sROP.

Evaluation of Risk Factors on ROP in the Netherlands

\section{Discussion}

In this second nationwide inventory on ROP and its risk factors (NEDROP-2), a ROP incidence of $28.3 \%$ was found. The incidence of sROP was $4.4 \%$. Risk factors found for overall ROP were GA, SGA, prolonged MV, and treatment with postnatal corticosteroids. For sROP, risk factors were 


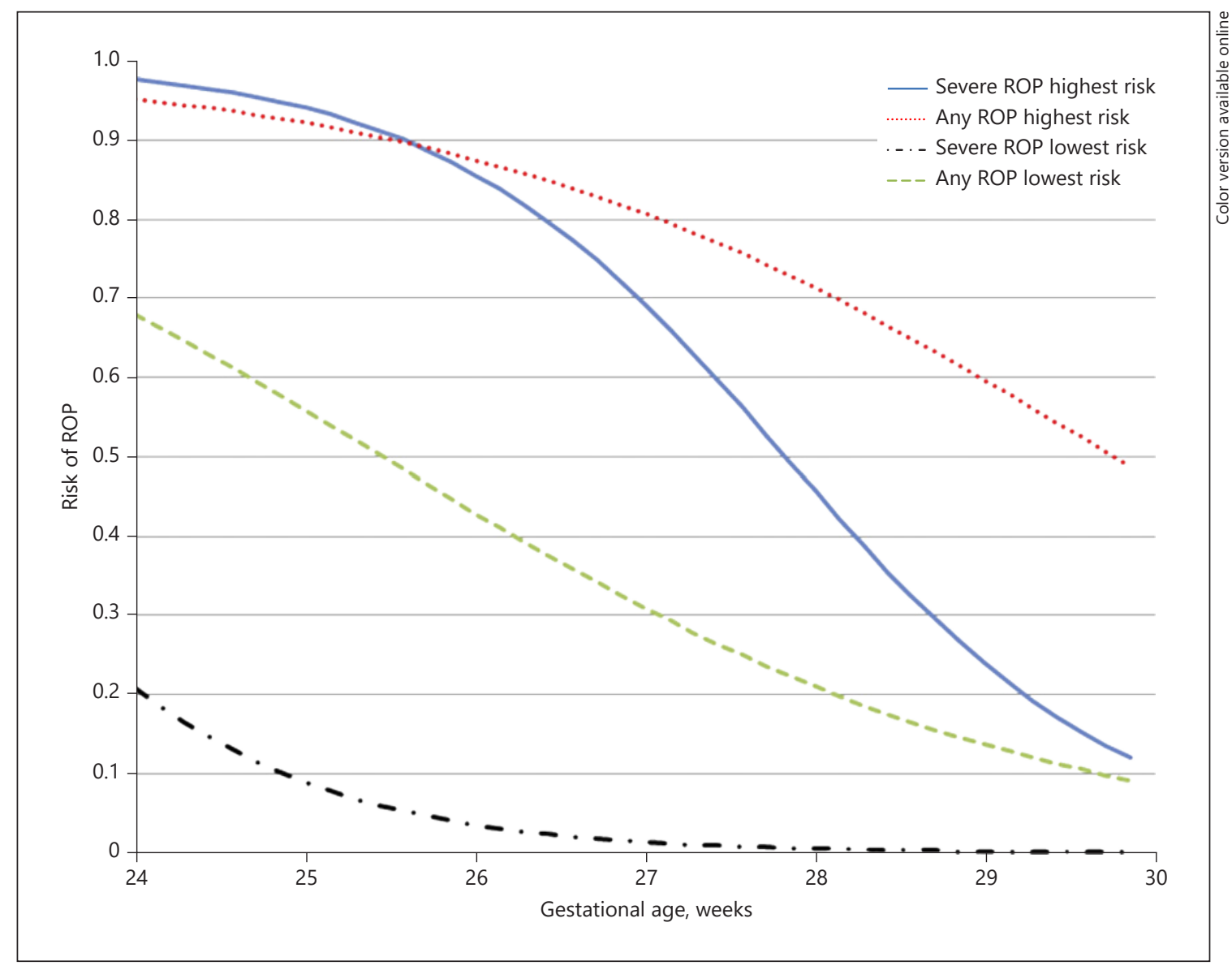

Fig. 2. Risk of (severe) ROP versus GA in weeks. Highest risk for any ROP defined as: exposure to all risk factors in Table 3 and lowest risk for any ROP: not exposed to any risk factors in Table 3. Highest risk for severe ROP: exposed to all risk factors in Table 4 and lowest risk for severe ROP: not exposed to risk factors in Table 4. ROP, retinopathy of prematurity; GA, gestational age.

Table 3. OR for any ROP, multivariable analysis, after adjusting for GA and SGA

\begin{tabular}{lcrr}
\hline Risk factors & OR & $95 \%$ CI & $p$ value \\
\hline Higher GA (weeks) & 0.59 & $0.54-0.66$ & $<0.001$ \\
SGA & 1.73 & $1.11-2.62$ & 0.014 \\
MV $>$ 7 days & 2.13 & $1.35-3.37$ & 0.001 \\
Postnatal corticosteroids & 2.57 & $1.44-4.66$ & 0.002 \\
\hline
\end{tabular}

ROP, retinopathy of prematurity; $\mathrm{OR}$, odds ratio; $\mathrm{CI}$, confidence interval; GA, gestational age; SGA, small for gestational age; $\mathrm{MV}$, mechanical ventilation.

GA, SGA, NEC, and postnatal corticosteroids. Prolonged MV was also associated with sROP, but postnatal corticosteroids had higher odds, and in our cohort, all infants who received corticosteroids had prolonged ventilation.
Table 4. OR for severe ROP, multivariable analysis, after adjusting for GA and SGA

\begin{tabular}{llll}
\hline Risk factors & OR & $95 \%$ CI & $p$ value \\
\hline Higher GA (weeks) & 0.37 & $0.27-0.50$ & $<0.001$ \\
SGA & 5.65 & $2.17-14.92$ & $<0.001$ \\
Perforated NEC & 7.55 & $2.29-24.48$ & $<0.001$ \\
Postnatal corticosteroids & 3.81 & $1.74-8.40$ & $<0.001$ \\
\hline
\end{tabular}

ROP, retinopathy of prematurity; OR, odds ratio; $\mathrm{CI}$, confidence interval; GA, gestational age; SGA, small for gestational age; NEC, necrotizing enterocolitis.

Overall ROP incidence in the NEDROP-1 was $21.9 \%$. Comparing the absolute ROP incidence of the current study to that of the NEDROP-1 study is difficult as inclusion criteria for ROP screening were narrowed since 
2013, which meant that infants with GA 30.0-32.0 weeks and/or with BW 1250-1,500 g without risk factors were no longer included, resulting in a lower denominator. Pediatricians reported 1,287 eligible infants in NEDROP-2, compared to 1,900 in NEDROP-1, a reduction of $32 \%$, which concords with the estimated $29 \%$ reduction after narrowing our screening inclusion criteria. Another explanation for fewer reported infants may be the lower number of live births in 2017. The percentage of infants of the reported population that could be coupled and screened was almost the same for both studies: 933/1,287 $72.5 \%$ (NEDROP-2) and 1,380/1,900 72.6\% (NEDROP-1). The higher incidence of ROP found in NEDROP-2 might be explained by more immature infants, fewer low-risk infants through narrowing of the inclusion criteria, and a lower birth number.

Compared to the NEDROP-1, the incidence of sROP was higher $(4.4 \%$ vs. $2.1 \%$ and $p<0.05)$, but also this difference should be interpreted with caution since the number of eligible infants differed and more immature infants were included. However, the incidence of sROP among all live births with GA $<32.0$ weeks registered in Perined was $41 / 1,452(2.8 \%)$ in 2017 and 29/1,602 (1.9\%) in 2009 $(p=0.06)$. A limitation to our study includes that 8 infants with sROP could not be coupled to the Perined database and were therefore excluded. If they would have enrolled, the increase between 2009 and 2017 would have reached statistical significance as in 2009 only 1 patient with sROP could not be coupled $(p=0.009)$. The results of another Dutch study, showing a doubling of treatments from 2013-2017 compared to 2010-2013, support this result [8]. Similar increases in sROP have been reported in Sweden, Denmark, and the UK [14-16].

Risk factors for ROP in NEDROP-2 were GA, SGA $(<-2 \mathrm{SD})$, prolonged $\mathrm{MV}$, and treatment with postnatal corticosteroids. GA and prolonged MV were also found as risk factors in NEDROP-1. In this latter study, the 95\% confidence interval for postnatal corticosteroids bordered on 1 , with a $p$ value of 0.08 . An explanation for postnatal corticosteroids reaching statistical significance in NEDROP-2 could be that since the change in our national treatment policy in 2010, more infants with GA $>24.0$ weeks, frequently needing postnatal corticosteroids, survive. Compared to NEDROP-1, iNO and prolonged stay on the NICU were no longer present as risk factors and female gender was no longer protective. An explanation may be that both iNO and LOS are strongly associated with MV, as in the Netherlands, iNO is almost only administered through MV and long-standing MV is only used in level III NICUs. In addition, LOS during the last

Evaluation of Risk Factors on ROP in the Netherlands decade may have been shortened by the expansion of level II + neonatal step-down units. Further, in NEDROP-2, there were more female infants with ROP $(31.9 \%)$ than male $(25.6 \%)$. Also, this may be the result of increased survival in extremely preterm female infants [17]. Since analysis of risk factors for sROP was not possible in NEDROP-1 because of the small number of infants with sROP, a comparison of risk factors is not possible. Finally, in the present dataset, information about prenatal corticosteroids was not available and could not be analyzed.

Our present risk factors are consistent with other recent studies in high-income countries. GA and BW are still the strongest known risk factors for (treatment requiring) ROP [18]. Both factors are related to the extent of immaturity of neural and vascular retinal development at birth. The lower GA and BW, the more profound the loss of growth factors normally provided by the intrauterine environment, the longer the exposure to adverse postnatal events and as a consequence of these, and the higher the retinal vulnerability to insult [1]. Razak and Faden [19] demonstrated that also smaller size for gestation was associated with increased odds of any ROP, sROP, and treated ROP. The retina of SGA babies has already intrauterine been exposed to neuroendocrine and metabolic adaptations which may increase the development of ROP. SGA infants are often treated with supplemental oxygen, a risk factor for ROP because of increased risk of respiratory distress syndrome, bronchopulmonary dysplasia, and NEC. They also have lower insulin-like growth factor-1 levels, causing an arrest in retinal vessel growth, resulting in phase 1 of ROP, and they have an increased prevalence of Frizzled-4 gene variation, a gene associated with increased risk of ROP [19].

Prolonged MV is among the most frequently identified risk factors for ROP [20]. In a Danish study, blood transfusion and MV were the only new risk factors to predict treatment-demanding ROP in addition to GA, SGA, multiple births, and male sex [21]. Prolonged MV is often associated with high percentages of supplemental oxygen and fluctuations in oxygen saturation levels.

The most recent Cochrane-study reported an increase in sROP after late systemic postnatal treatment with corticosteroids ( $>7$ days) but no increase in blindness [22]. In our cohort, we found an increased risk for postnatal corticosteroids for any ROP as well as sROP. An explanation for this may be that in the Netherlands, most infants receive late corticosteroid treatment as early treatment is associated with gastrointestinal bleeding, perforation, and cerebral palsy. NEC was an additional risk factor for ROP in the predicting model of Gonski [23]. The exact relationship between NEC and ROP is unclear. Animal 
studies showed that systemic inflammation affects retinal angiogenesis [18]. From our estimated risk model (Fig. 2), it is clear that for Dutch infants with a GA $<26.0$ weeks with a high-risk profile, the risk to develop ROP or sROP is almost equal. This means that if such an infant develops ROP, the chance is extremely high that it will also develop into severe, treatment-requiring ROP. This may be explained by the fact that many of these infants have a complicated clinical course. Our risk model may be important for future interventional studies and can also be a help to explain the risk of ROP to parents. Limitations of our study are loss of patients after merging, risk of bias as a result of not being able to completely match the databases, and restricted presence of risk factors present in the Perined database, especially the known influence of oxygen fluctuations on the development of ROP that we were not able to measure. Since 2013, the Dutch screening guideline for ROP has been adjusted, based on the results of NEDROP-1. The most important aim of screening is that infants with sROP, needing treatment, are timely detected. The present study, which indicates GA, SGA ( $p<$ -2 SD), NEC, and postnatal corticosteroids as risk factors for sROP, with the available data did not indicate new factors apart from those already included in our present guideline. Severe ROP in NEDROP-2 was only found in infants with GA $<30.0$ weeks as to 5 infants with GA 30.0 32.0 weeks in NEDROP-1. Whether our guideline can be adjusted in the future needs more evaluation. To answer this, a study on cost-effectiveness of screening in the Netherlands is currently being conducted.

In conclusion, severe ROP was diagnosed more frequently since the last inventory on ROP. Risk factors for severe ROP were GA, SGA, NEC, and postnatal corticosteroids. All these factors are included in our screening guideline, emphasizing the benefits of our risk-based guideline.

\section{Acknowledgements}

We thank all participating ophthalmologists, neonatologists, and pediatricians for their contribution.

\section{Statement of Ethics}

This study was initiated and coordinated by the Leiden University Medical Center. Approval was obtained by the local medical Ethical Committee. In the Netherlands, neonatal data are recorded in a national perinatal register called Perined. For this registry, parents have to give their approval. As ROP is part of Perined, informed consent for the present study was waived.

\section{Conflict of Interest Statement}

The authors have no conflicts of interest to declare.

\section{Funding Sources}

This study was supported by a grant from ODAS Foundation, Delft. Project number 2016-02 NEDROP-2.

\section{Author Contributions}

Research design was done by K.T., N.S., and J.T. All the authors provided data for analysis. Acquisition and analysis of data were performed by K.T. and S.K. The authors F.G. and K.T. were responsible for statistical analysis. The article was written by K.T., J.T. F.G., and N.S. and was critically revised and approved for publication by P.A., P.D., F.D., J.H., S.K., R.K., E.W., J.L., C.M., F.S., K.S., M.T., and R.W.

\section{References}

1 Hellstrom A, Smith LE, Dammann O. Retinopathy of prematurity. Lancet. 2013; 382(9902):1445-57.

2 Blencowe H, Lawn JE, Vazquez T, Fielder A, Gilbert C. Preterm-associated visual impairment and estimates of retinopathy of prematurity at regional and global levels for 2010 . Pediatr Res. 2013;74(Suppl 1):35-49.

3 Tavassoli S, Wach R, Haynes R, Markham R, Williams C. Estimate of incidence of ROP requiring treatment in extreme preterms and impact on service-7 year review in tertiary unit. Eye. 2019;33(5):845-9.
4 Kim SJ, Port AD, Swan R, Campbell JP, Chan RVP, Chiang MF. Retinopathy of prematurity: a review of risk factors and their clinical significance. Surv Ophthalmol. 2018;63(5): 618-37.

5 van Sorge AJ, Termote JU, Kerkhoff FT, van Rijn LJ, Simonsz HJ, Peer PG, et al. Nationwide inventory of risk factors for retinopathy of prematurity in the Netherlands. J Pediatr. 2014;164(3):494-8.e1.

6 van Sorge AJ, Schalij-Delfos NE, Kerkhoff FT, van Rijn LJ, van Hillegersberg JL, van Liempt IL, et al. Reduction in screening for retinopa- thy of prematurity through risk factor adjusted inclusion criteria. Br J Ophthalmol. 2013; 97(9):1143-7.

7 Saugstad OD, Aune D. Optimal oxygenation of extremely low birth weight infants: a metaanalysis and systematic review of the oxygen saturation target studies. Neonatology. 2014; 105(1):55-63.

8 Trzcionkowska K, Vehmeijer W, Kerkhoff FT, Bauer NJC, Bennebroek CAM, Dijk PH, et al. Increase in treatment of retinopathy of prematurity in the Netherlands from 2010 to 2017. Acta Ophthalmol. 2021;99(1):97-103. 
9 Hoftiezer L, Hof MHP, Dijs-Elsinga J, Hogeveen M, Hukkelhoven CWPM, van Lingen RA. From population reference to national standard: new and improved birthweight charts. Am J Obstet Gynecol. 2019;220(4): 383.e1-e17.

10 Papile LA, Burstein J, Burstein R, Koffler H. Incidence and evolution of subependymal and intraventricular hemorrhage: a study of infants with birth weights less than 1,500 gm. J Pediatr. 1978;92(4):529-34.

11 de Vries LS, Eken P, Dubowitz LM. The spectrum of leukomalacia using cranial ultrasound. Behav Brain Res. 1992;49(1):1-6.

12 International Committee for the Classification of Retinopathy of Prematurity. The international classification of retinopathy of prematurity revisited. Arch Ophthalmol. 2005; 123(7):991-9.

13 Good WV. Final results of the Early Treatment for Retinopathy of Prematurity (ETROP) randomized trial. Trans Am Ophthalmol Soc. 2004; 102:233-50; discussion 4850.
14 Holmström G, Tornqvist $\mathrm{K}$, Al-Hawasi A, Nilsson Å, Wallin A, Hellström A. Increased frequency of retinopathy of prematurity over the last decade and significant regional differences. Acta Ophthalmol. 2018;96(2):142-8.

15 Slidsborg C, Olesen HB, Jensen PK, Jensen H, Nissen KR, Greisen G, et al. Treatment for retinopathy of prematurity in Denmark in a ten-year period (1996 2005): is the incidence increasing? Pediatrics. 2008;121(1):97-105.

16 Hameed B, Shyamanur K, Kotecha S, Manktelow BN, Woodruff G, Draper ES, et al. Trends in the incidence of severe retinopathy of prematurity in a geographically defined population over a 10-year period. Pediatrics. 2004;113(6):1653-7.

17 Anderson JG, Baer RJ, Partridge JC, Kuppermann M, Franck LS, Rand L, et al. Survival and major morbidity of extremely preterm infants: a population-based study. Pediatrics. 2016;138(1):e20154434.

18 Kim SJ, Port AD, Swan R, Campbell JP, Chan RVP, Chiang MF. Retinopathy of prematurity: a review of risk factors and their clinical significance. Surv Ophthalmol. 2018;63(5): 618-37.
19 Razak A, Faden M. Association of small for gestational age with retinopathy of prematurity: a systematic review and meta-analysis. Arch Dis Child Fetal Neonatal Ed. 2020; 105(3):270-8.

20 Chaves-Samaniego MJ, García Castejón M, Chaves-Samaniego MC, Solans Perez Larraya A, Ortega Molina JM, Muñoz Hoyos A, et al. Risk calculator for retinopathy of prematurity requiring treatment. Front Pediatr. 2020;8: 529639.

21 Slidsborg C, Jensen A, Forman JL, Rasmussen S, Bangsgaard R, Fledelius HC, et al. Neonatal risk factors for treatment-demanding retinopathy of prematurity: a Danish national study. Ophthalmology. 2016;123(4):796-803.

22 Doyle LW, Cheong JL, Ehrenkranz RA, Halliday HL. Late ( $>7$ days) systemic postnatal corticosteroids for prevention of bronchopulmonary dysplasia in preterm infants. Cochrane Database Syst Rev. 2017;10:Cd001145.

23 Gonski S, Hupp SR, Cotten CM, Clark RH, Laughon M, Watt K, et al. Risk of development of treated retinopathy of prematurity in very low birth weight infants. J Perinatol. 2019;39(11):1562-8. 